\section{CORRECTION}

View Article Online

View Journal I View Issue

\section{D) Check for updates}

Cite this: Nanoscale, 2021, 13, 4685

DOI: $10.1039 / \mathrm{d} 1 \mathrm{nr} 90031 \mathrm{e}$

rsc.li/nanoscale

\title{
Correction: Electronic conductance and thermopower of single-molecule junctions of oligo(phenyleneethynylene) derivatives
}

\author{
Hervé Dekkiche, ${ }^{a}$ Andrea Gemma, ${ }^{b}$ Fatemeh Tabatabaei, ${ }^{c}$ Andrei S. Batsanov, ${ }^{a}$ \\ Thomas Niehaus, ${ }^{\star c}$ Bernd Gotsmann ${ }^{* b}$ and Martin R. Bryce*a
}

Correction for 'Electronic conductance and thermopower of single-molecule junctions of oligo(phenyleneethynylene) derivatives' by Hervé Dekkiche et al., Nanoscale, 2020, 12, 18908-18917, DOI: 10.1039/ D0NR04413J.

The authors have discovered an error that affects some of the numerical results shown in the original article. In the transport simulations the lead self-energy was not sufficiently converged, which resulted in an underestimated transmission close to the Fermi energy. As such, a $10 \times 10 \times 10$ instead of a $2 \times 1 \times 1$ (original article) $k$-point sampling has been used in the construction of the lead self-energy. The updated transmission plot (Fig. 7) and corrected values for conductance and Seebeck coefficients (Table 1) are displayed below. These errors do not affect any of the experimental results and discussion or conclusions reported in the paper, only the display of Fig. 7 and Table 1.

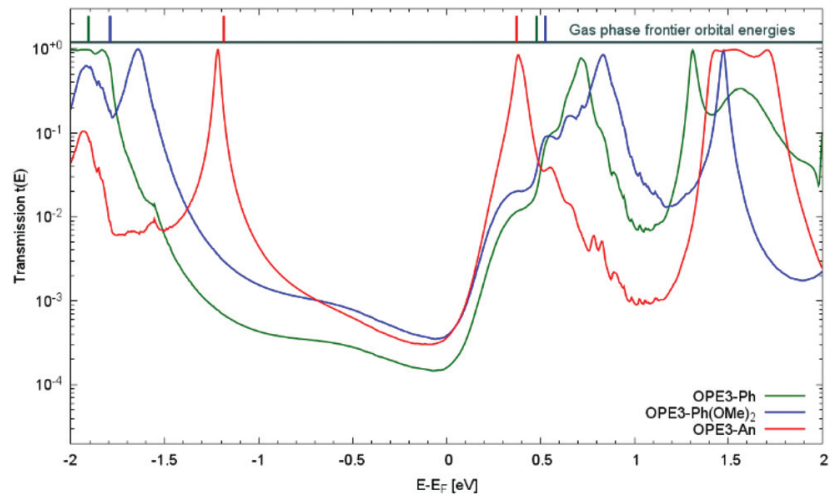

Fig. 7 DFTB transmission function $t(E)$ for three OPE3 derivatives. For illustrative purposes the HOMO and LUMO energies of the isolated molecules in the gas phase are given at the top of the figure. To account for the Fermi level alignment upon contact formation, the gas phase energies have been shifted by $\Delta=\mathrm{Md}-\mathrm{Mg}$, where Md and Mg denote the HOMO/LUMO mid gap energies for the device and gas phase, respectively. We obtain $\triangle \mathrm{OPE} 3-\mathrm{Ph}=-1.45 \mathrm{eV}, \triangle \mathrm{OPE3}-\mathrm{Ph}(\mathrm{OMe})_{2}=-1.44 \mathrm{eV}$ and $\triangle \mathrm{OPE3}-\mathrm{An}=-1.00 \mathrm{eV}$. The sign of these shifts is consistent with a charge transfer of electrons from the molecule to the electrode through formation of dative bonds.

${ }^{a}$ Department of Chemistry, Durham University, Durham, DH1 3LE, UK. E-mail: m.r.bryce@durham.ac.uk

${ }^{b}$ IBM Research-Zurich, Rueschlikon 8803, Switzerland. E-mail: bgo@zurich.ibm.com

${ }^{c}$ Université Claude Bernard Lyon 1, CNRS, Institut Lumière Matière, Villeurbanne, France. E-mail: thomas.niehaus@univ-lyon1.fr 
Table 1 Experimental and theoretical values for the conductance $G$ and Seebeck coefficients $S$ at room temperature $(T=295 \mathrm{~K})$ for different $O P E 3$ derivatives obtained in this study. For each molecule, $G_{\text {exp }}$ is the peak-value of the distribution used to build the 2D histogram (first column of Fig. 4) From the experimental value of $S$, the contribution of the copper leads $\left(1.8 \mu \mathrm{V} \mathrm{K}{ }^{-1}\right.$ ) was subtracted (see ESI $\dagger$ )

\begin{tabular}{lllll}
\hline & $G_{\exp }\left[10^{-4} \mathrm{G}_{0}\right]$ & $G_{\text {theo }}\left[10^{-4} \mathrm{G}_{0}\right]$ & $S_{\text {exp }}\left[\mu \mathrm{V} \mathrm{K} \mathrm{K}^{-1}\right]$ & $S_{\text {theo }}\left[\mu \mathrm{V} \mathrm{K}{ }^{-1}\right]$ \\
\hline OPE3-Ph & 0.92 & 1.60 & $-11.4 \pm 0.5$ & -25.6 \\
OPE3-Ph(OMe) $)_{2}$ & 2.03 & 3.86 & $-7.9 \pm 1.3$ & -27.0 \\
OPE3-An & 1.82 & 3.64 & $-8.7 \pm 1.6$ & -37.1
\end{tabular}

The Royal Society of Chemistry apologises for these errors and any consequent inconvenience to authors and readers. 